\title{
Derivation and Analysis of Dynamic Handwriting Features as Clinical Markers of Parkinson's Disease
}

\author{
Christine Poon \\ The University of Sydney \\ christine.poon@sydney.edu.au

Kelvin Tsoi
Chinese University of Hong
Kong
kelvintsoi@,cuhk.edu.hk

\author{
Niku Gorji \\ The University of Sydney \\ niku.gorji@sydney.edu.au \\ S.T. Boris Choy \\ The University of Sydney \\ boris.choy@sydney.edu.au \\ Simon Poon \\ The University of Sydney \\ Simon.poon@sydney.edu.au
}

\author{
Mark Latt \\ The University of Sydney \\ mark.latt@sydney.edu.au \\ Clement Loy \\ The University of Sydney \\ niku.gorji@sydney.edu.au
}

\begin{abstract}
Parkinson's Disease (PD) is a complex neurodegenerative disorder that is challenging to diagnose. Recent research has demonstrated predictive value in the analysis of dynamic handwriting features for detecting $P D$, however, consensus on clinicallyuseful features is yet to be reached. Here we explore and evaluate secondary kinematic handwriting features hypothesized to be diagnostically relevant to Parkinson's Disease using a publicly-available Spiral Drawing Test PD dataset. Univariate and multivariate analysis was performed on derived features. Classification outcome was determined using logistic regression models with 10 -fold cross validation. Feature correlation was based on model specificity and sensitivity. Variations in grip angle, instantaneous acceleration and pressure indices were found to have high predictive potential as clinical markers of $P D$, with combined classification accuracy of above $90 \%$. Our results show that the significance of secondary handwriting features and recommend the feature expansion step for hypothesis generation, comparative evaluation of test types and improved classification accuracy.
\end{abstract}

\section{Introduction}

Parkinson's Disease (PD) is one of the most prevalent neurodegenerative disorders, affecting approximately $1 \%$ of the population aged over 65 years [1]. PD is a progressive disorder that primarily affects the motor system, characterized by tremor, rigidity, slowness of movement (bradykinesia), loss of fine motor control, postural instability and latter-stage cognitive decline [2]. As handwriting is a complex process involving motor planning, programming, sequencing, initiation and execution [3], impaired handwriting (dysgraphia) is one of the earliest presentations of disrupted motor control with PD [2, 4]. As there is currently no cure for the condition, early and accurate diagnosis is critical for effective management of disease progression through interventive therapies. However, the current diagnostic regime relies on subjective clinical examination with observations scored on rating scales e.g. Unified Parkinson's Disease Rating Scale (UPDRS) [5] or the Hoehn and Yahr scale (H\&Y) [6], leading to diagnostic scores that can vary depend on the experience and interpretation of the treating neurologist [7]. The rating scale system is insensitive to subtle early manifestations of neurodegeneration and has a misdiagnosis rate of up to $25 \%$ [8]. More objective means of assessing symptoms are required for earlier detection, improved diagnostic accuracy and therefore patient outcome.

Advances in digitized handwriting technologies have enabled measurement, quantification and analysis of kinematic handwriting process features, offering the potential for earlier detection and more accurate diagnosis of PD. This application represents a cuttingedge intersection of neuroscience, data analytics, artificial intelligence and kinesiology, and has enjoyed active development. Generally, PD handwriting and drawing test tasks are recorded using a digitized tablet and/or stylus and stored as a number of raw variables, most fundamentally: $\mathrm{x}, \mathrm{y}$ and $\mathrm{z}$ coordinates, time and 
relative pressure. Grip angle may also be recorded depending on the capabilities of the data collection platform and software. From these raw variables, features of both the final writing output and kinematic handwriting process are calculated for analysis and classification.

While progress has been impressive [9-11], consensus on clinically significant handwriting process features for diagnosis of PD is yet to be reached [12, 13]. Features reported to hold high statistical correlation with PD vary depending on the study design, drawing tasks, data collection tools, statistical models and classifier types, the selection of which intrinsically determines the types of data, quality and depth of insight obtainable. The lack of standardization between studies to date do not allow for meaningful comparison and evaluation.

In this study, we apply a combination of a topdown and bottom-up design approach to progress towards a standardizable digitized diagnostic support tool for PD. We propose that in order to develop the most accurate PD classification model possible, it is essential to extract and evaluate all potentially informative handwriting features from a 'typical' raw dataset regardless of recording platform. Furthermore, as handwriting is a complex, time-dependent sequence of preprogrammed movement, we hypothesize that analysis of the change in commonly reported features with time may provide further insight and predictive correlation to the physiological neuropathology of PD. We also hypothesize that broadened analysis of kinematic handwriting features may unlock informative trends to allow for objective evaluation and comparison of existing test designs.

To address this gap in current knowledge, we conducted feature expansion and analysis on a PD spiral drawing dataset. We followed an integrated stepwise approach: (i) univariate analysis to individually identify the most relevant handwriting features for accurate differentiation of PD patients from healthy controls, and (ii) using a stepwise backward elimination approach to develop a multivariate combinative model based on the most significant set of handwriting features with potential application as an objective clinical screening or diagnostic tool. The following sections present a brief overview of handwriting analysis, our approach and findings. The physiological correlation of statistically significant features with PD and comparison of the spiral drawing tasks analyzed will also be presented along with perspectives for further feature-driven development.

\section{Handwriting Analysis in Parkinson's Disease: An Overview}

A spectrum of impaired handwriting (dysgraphia) is well documented to be clinical hallmarks of PD, including micrographia (smaller than normal handwriting), slower writing speed and jerk due to tremor. Traditionally, clinical assessment is based on observation of both the handwriting process and static output of drawing and writing tasks designed to provide a tangible measure of motor-cognitive function by the level of writing proficiency.

\section{A Spiral measures}
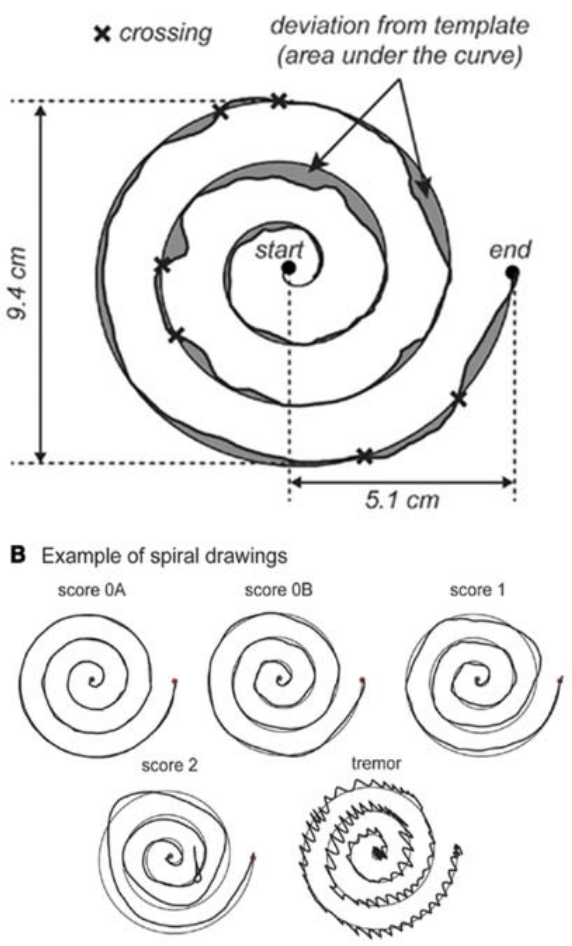

Figure 1. Examples of spiral test analysis and clinical scoring, (source: Hoogendam et al. [31])

Advances in digitized pen and tablet technologies have enabled the scientific measurement and analysis of static and dynamic handwriting metrics (graphonomics). This capability augments the depth of insight that can be obtained from writing and drawing tasks and potentially unlocks a wealth of previously unmeasurable information. Research to date has focused on digitizing established drawing tasks for assessing cognitive impairment, including: signature writing [11, 14], writing short phrases [15], pentagon copying [16-18], clock drawing [19], spiral drawing [20-22], circles and cursive looping [23, 24]. Of these, 
writing words and phrases containing repetitions of cursive ' $l$ ' and ' $e$ ' have been commonly reported [2527]. However, writing is influenced by culture, penmanship training, level of education and linguistic ability [28]. Drawing tasks eliminates influential training bias and therefore allows for more universal application. The standard spiral drawing test (SST) is commonly used for evaluating motor performance and tremor in PD [12, 24, 29-31].

In this test, patients trace an Archimedean spiral that has been pre-templated on a programmable pressure-sensitive graphics tablet; the degree of accuracy of following the template provides quantifiable assessment of tremor and handwriting impairment. A piece of paper may be overlaid to provide instant visual feedback. Data is stored as raw variables and processed offline. Analytical measures of a standard Archimedean spiral drawing test and examples of PD scoring are shown in Figure 1. The dynamic spiral test (DST) is a progressive variation of the SST proposed by Isenkul et al that involves tracing a blinking spiral template. The temporal component introduces another dimension of cognitive challenge which allows for correlative assessment of visualmotor feedback.

\subsection{Data Acquisition Tools}

Digitization tablets in this area of research has been dominated by WACOM tablets (Wacom Technology Corporation, Portland, Oregon U.S.A), particularly the Intuos Pro series as they offer the highest spatial and temporal resolutions among their competitors [32]. Outputs of drawing tasks are typically recorded on custom software as raw variables, e.g. cartesian coordinates of a pen/stylus point, pressure and time, which are then processed for analysis.

Various C, R and Matlab-based software systems have been developed to calculate graphic and kinematic handwriting features; most are custom and study-specific with the exception of MovAlyzeR and ComPET $[25,33]$.

\subsection{Handwriting Feature Analysis}

Typical features that have been investigated in literature to date can be considered to fall under two categories: graphic and kinematic. Graphic handwriting features describe static measures of the drawing output such as geometry, whereas kinematic features pertain to measures of dynamic handwriting processes. These are summarized in Table 1 and Table 2 respectively.

\section{Methods}

The focus of this study is to investigate whether secondary dynamic handwriting features in addition to those graphic features summarized in Table 1 hold predictive classification value, as well as whether they formulate informative indices for evaluating and comparing cognitive drawing tasks designed for diagnosing Parkinson's Disease.

The steps of our analysis are summarized in Figure 2 and are presented in the following sections.

Table 1. Graphic handwriting features relevant to diagnosis of PD and other neuro

\begin{tabular}{|c|c|}
\hline \multicolumn{2}{|l|}{ Graphic } \\
\hline Feature & Description \\
\hline Stroke length & $\begin{array}{l}\text { Absolute magnitude of } \text { on-surface } \\
\text { movement between two successive } \\
\text { pen-lifts }\end{array}$ \\
\hline Stroke height & $\begin{array}{l}\text { Absolute magnitude between the } \\
\text { highest and lowest } y \text {-value positions of } \\
\text { the stroke }\end{array}$ \\
\hline Stroke width & $\begin{array}{l}\text { Absolute magnitude between the } \\
\text { highest and lowest } x \text {-value positions of } \\
\text { the stroke }\end{array}$ \\
\hline Stroke duration & Total time taken draw a stroke \\
\hline & $\begin{array}{l}\text { Pressure recorded by a digitized pen or } \\
\text { tablet, expressed in unscaled units }\end{array}$ \\
\hline $\begin{array}{r}\text { features rele } \\
\text { neu }\end{array}$ & $\begin{array}{l}\text { atic handwriting } \\
\text { s of PD and other } \\
\text { isorders }\end{array}$ \\
\hline Kinematic & \\
\hline Feature & Description \\
\hline Stroke speed & $\begin{array}{l}\text { Stroke length divided by stroke } \\
\text { duration }(\mathrm{mm} / \mathrm{s})\end{array}$ \\
\hline Speed* & $\begin{array}{l}\text { Absolute magnitude of velocity } \\
(\mathrm{mm} / \mathrm{s})\end{array}$ \\
\hline Velocity* & $\begin{array}{l}\text { Rate of directional change of pen } \\
\text { position with time }(\mathrm{mm} / \mathrm{s})\end{array}$ \\
\hline Acceleration* & $\begin{array}{l}\text { Rate of change of pen velocity with } \\
\text { time }\left(\mathrm{mm} / \mathrm{s}^{2}\right)\end{array}$ \\
\hline Jerk & $\begin{array}{l}\text { Rate of change of pen acceleration } \\
\text { with time }\left(\mathrm{mm} / \mathrm{s}^{3}\right)\end{array}$ \\
\hline Grip angle & $\begin{array}{l}\text { Angle the pen is held relative to the } \\
\text { writing surface }\end{array}$ \\
\hline In-air time & $\begin{array}{l}\text { Time the pen is lifted off the writing } \\
\text { surface during the task }\end{array}$ \\
\hline Contact time & $\begin{array}{l}\text { Duration of pen contacting the writing } \\
\text { surface (normalized over total task } \\
\text { duration) }\end{array}$ \\
\hline $\begin{array}{l}\text { Change in } \\
\text { pressure }\end{array}$ & Variation in applied pressure \\
\hline
\end{tabular}




\subsection{Data acquisition \& preprocessing}

Latent feature derivation and analysis was conducted using Isenkul et al's PD study dataset
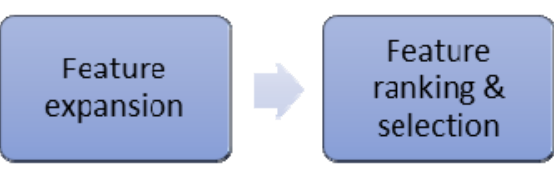

containing the raw experimental drawing test data of a sample of 40 patients (25 PD patients and 15 healthy controls) [34].

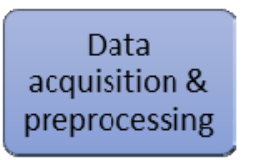

Figure 2. An optimized approach to creating a classifier system for PD from raw graphonomics data

Table 3. Primary and secondary handwriting features derived from raw data variables

\begin{tabular}{|c|c|c|}
\hline \multicolumn{3}{|l|}{ Primary Features } \\
\hline Feature & Label & Description/Formula \\
\hline Width (total) & Width & Maximum $\mathrm{X}$ co-ordinate minus minimum $\mathrm{X}$ co-ordinate \\
\hline Height (total) & Height & Maximum Y co-ordinate minus minimum Y co-ordinate \\
\hline Length & Length & $\begin{array}{l}\text { Total length of the drawing, given by } \Sigma \sqrt{(x 1-x 0)^{2}+(y 1-y 0)^{2}} \text { with } \\
\text { continuous drawing i.e. } z=0\end{array}$ \\
\hline Speed & Velocity & Length $\div$ total drawing time $(\mathrm{V}=\mathrm{L} / \mathrm{T})$ \\
\hline $\begin{array}{l}\text { Peak instantaneous } \\
\text { speed }\end{array}$ & PIV & Highest speed recorded at any time point $\left(\mathrm{V}_{\mathrm{i}}=\mathrm{L}_{\mathrm{i}} / \mathrm{T}_{\mathrm{i}}\right)$ \\
\hline $\begin{array}{l}\text { Peak instantaneous } \\
\text { acceleration }\end{array}$ & PIA & Highest acceleration recorded at any time point $\left(A_{i}=V_{i} / T_{i}\right)$ \\
\hline \multicolumn{3}{|l|}{ Secondary Features } \\
\hline Feature & Label & Description/Formula \\
\hline Grip angle (Mean) & GripAngleMean & Average of grip angle values for the entire drawing task \\
\hline Grip angle (SD) & GripAngleSD & Standard deviation of grip angle values for the entire drawing task \\
\hline Pressure (Mean) & PressureMean & Average of recorded pressure values for the entire task \\
\hline Pressure (SD) & PressureSD & Standard deviation of recorded pressure values for the entire task \\
\hline $\begin{array}{l}\text { Positive pressure } \\
\text { change (Mean) }\end{array}$ & PCAvgPos & $\begin{array}{l}\text { Average increase in pressure between two time points, given by } p(i+1)-p(i) / \\
t(i+1)-t(i) \text { where } p(i+1)>p(i)\end{array}$ \\
\hline $\begin{array}{l}\text { Positive pressure } \\
\text { change (SD) }\end{array}$ & PCSDPos & $\begin{array}{l}\text { Standard deviation of increase in pressure between two time points, given by } \\
p(i+1)-p(i) / t(i+1)-t(i) \text { where } p(i+1)>p(i)\end{array}$ \\
\hline $\begin{array}{l}\text { Maximum positive } \\
\text { pressure change }\end{array}$ & PCMax & $\begin{array}{l}\text { Maximum increase in handwriting pressure between two time points, given by } \\
\operatorname{Max}[p(i+1)-p(i) / t(i+1)-t(i) \text { for } p(i+1)>p(i)]\end{array}$ \\
\hline $\begin{array}{l}\text { Negative pressure } \\
\text { change (mean) }\end{array}$ & PCAvgNeg & $\begin{array}{l}\text { Average decrease in pressure between two time points, given by } p(i+1)-p(i) / \\
t(i+1)-t(i) \text { where } p(i+1)<p(i)\end{array}$ \\
\hline $\begin{array}{l}\text { Negative pressure } \\
\text { change (SD) }\end{array}$ & PCSDNeg & $\begin{array}{l}\text { Standard deviation of decrease in pressure between two time points, given by } \\
\mathrm{p}(\mathrm{i}+1)-\mathrm{p}(\mathrm{i}) / \mathrm{t}(\mathrm{i}+1)-\mathrm{t}(\mathrm{i}) \text { where } \mathrm{p}(\mathrm{i}+1)<\mathrm{p}(\mathrm{i})\end{array}$ \\
\hline $\begin{array}{l}\text { Maximum negative } \\
\text { pressure change }\end{array}$ & PCMin & $\begin{array}{l}\text { Maximum reduction in handwriting pressure between two time points, given by } \\
\operatorname{Max}[\mathrm{p}(\mathrm{i}+1)-\mathrm{p}(\mathrm{i}) / \mathrm{t}(\mathrm{i}+1)-\mathrm{t}(\mathrm{i}) \text { for } \mathrm{p}(\mathrm{i}+1)<\mathrm{p}(\mathrm{i})]\end{array}$ \\
\hline
\end{tabular}

*feature values calculated from Dynamic Spiral Test dataset variables, which are non-unitized except for time

Each study participant performed three separate drawing tasks based on the spiral drawing: (i) Static
Spiral Test which involves drawing on top of an Archimedes spiral template programmed into the 
recording graphics tablet (Wacom Cintiq 12WX), (ii) Dynamic Spiral Test where the spiral template alternately appears and disappears at specific time intervals while the subject attempts to trace the spiral, and (iii) the circular motion test in which subjects draw circles around a central red point.

The raw dataset contains 6 handwriting variables for each test type: $\mathrm{x}$ position, $\mathrm{y}$ position, $\mathrm{z}$ position, pressure, grip angle and time, recorded at a sampling rate of 7 millisecond (interval) on custom software. As the DST uniquely explores an additional memorybased cognitive dimension compared to the SST, the datasets for both the static and dynamic spiral drawing experimental groups were analyzed in our preliminary study for comparison.

\subsection{Feature derivation $\&$ expansion phase}

Given that the dynamic spiral test is a progression of the standard spiral drawing test, it can be considered as a new test type that potentially holds new informative kinematic metrics relevant to $\mathrm{PD}$, and which therefore necessitates additional metrics to evaluate. Furthermore, we hypothesize that the change in kinematic features with time may provide informative correlation with physiological and clinical presentations of $\mathrm{PD}$ in addition to the features commonly investigated (Table 1 and Table 2).

Our study purposes to explore both primary features i.e. those which are commonly derived from raw data variables, and secondary handwriting features which provide a measure of 'submovements', i.e. the subtle change of kinematic features with time. This work represents the first reported exploration of such handwriting features, which we hypothesize to hold clinical relevance and may allow more sensitive detection of PD. Therefore, sixteen features were derived from the six raw numerical variables in the SST and DST datasets respectively (Table 3). The sixteen handwriting features were then screened for potential redundancy and ranked to determine the highest statistical correlation/classification accuracy.

\subsection{Feature Ranking \& Selection phase}

Univariate logistic regression with 10 -fold crossvalidation was performed on each of the 16 derived handwriting features according to the known condition outcomes: (PD) and control (healthy). The features for each test type (SST and DST) were then ranked from most correlative to least based on precision and recall measures as presented in Table 4 and Table 5 . Weighted average ROC (model fit) was also considered. For features to be considered statistically significant, their precision and recall values must both exceed a threshold of 0.375 for the control group (15 out of a sample of 40), and 0.625 for PD ( 25 out of 40 ). The most significant features for each test type are highlighted in the respective tables.

From univariate analysis of each feature derived from SST raw data (shown in Table 4), the most significant SST handwriting features are: peak instantaneous acceleration (PIA), maximum increase and reduction in pressure (PCMax and PCMin), standard deviation in pressure (PSD) and standard deviation of grip angle (GASD).

Table 4. Results of univariate feature ranking results for SST based on precision and recall

\begin{tabular}{|l|rr|rl|l|}
\hline SST & \multicolumn{3}{|l|}{ Precision } & Recall & ROC \\
\cline { 1 - 5 } Variable & Control & PD & Control & PD & \\
\hline PIA & 0.688 & 0.833 & 0.792 & 0.792 & 0.792 \\
PCMin & 0.706 & 0.87 & 0.861 & 0.861 & 0.861 \\
PSD & 0.692 & 0.778 & 0.741 & 0.741 & 0.741 \\
PCMax & 0.667 & 0.75 & 0.741 & 0.741 & 0.741 \\
GASD & 0.667 & 0.75 & 0.752 & 0.752 & 0.752 \\
PMean & 0.667 & 0.8 & 0.667 & 0.667 & 0.667 \\
PCSDPos & 0.571 & 0.667 & 0.699 & 0.699 & 0.699 \\
PCAvgPos & 0.5 & 0.667 & 0.712 & 0.712 & 0.712 \\
PCSDNeg & 0.5 & 0.647 & 0.752 & 0.752 & 0.752 \\
PCAvgNeg & 0.4 & 0.629 & 0.643 & 0.643 & 0.643 \\
Height & 0.333 & 0.622 & 0.597 & 0.597 & 0.597 \\
Width & 0.2 & 0.6 & 0.713 & 0.713 & 0.713 \\
Length & 0 & 0.615 & 0.245 & 0.245 & 0.245 \\
Velocity & 0 & 0.615 & 0.328 & 0.328 & 0.328 \\
GAMean & 0 & 0.625 & 0.232 & 0.232 & 0.232 \\
PIV & 0 & 0.625 & 0.411 & 0.411 & 0.411 \\
\cline { 1 - 4 } & & & & &
\end{tabular}

Table 5. Results of univariate feature ranking for DST based on precision and recall

\begin{tabular}{|c|c|c|c|c|c|}
\hline DST & \multicolumn{2}{|c|}{ Precision } & \multicolumn{2}{|l|}{ Recall } & \multirow[b]{2}{*}{ ROC } \\
\hline Variable & Control & PD & Control & $\mathrm{PD}$ & \\
\hline GASD & 0.667 & 0.8 & 0.667 & 0.8 & 0.813 \\
\hline PIA & 0.75 & 0.786 & 0.6 & 0.88 & 0.84 \\
\hline PMean & 0.643 & 0.769 & 0.6 & 0.8 & 0.635 \\
\hline PCMax & 0.778 & 0.742 & 0.467 & 0.92 & 0.765 \\
\hline Height & 0.5 & 0.667 & 0.333 & 0.8 & 0.68 \\
\hline PSD & 0.444 & 0.645 & 0.267 & 0.8 & 0.685 \\
\hline PCSDPos & 0.5 & 0.632 & 0.067 & 0.96 & 0.701 \\
\hline PCMin & 0 & 0.615 & 0 & 0.96 & 0.483 \\
\hline PCAvgNeg & 0 & 0.615 & 0 & 0.96 & 0.259 \\
\hline PCAvgPos & 0 & 0.615 & 0 & 0.96 & 0.667 \\
\hline PCSDNeg & 0 & 0.615 & 0 & 0.96 & 0.283 \\
\hline Velocity & 0 & 0.615 & 0 & 0.96 & 0.312 \\
\hline Length & 0 & 0.605 & 0 & 0.92 & 0.341 \\
\hline Width & 0 & 0.595 & 0 & 0.88 & 0.456 \\
\hline GAMean & 0 & 0.625 & 0 & 1 & 0.309 \\
\hline PIV & 0 & 0.625 & 0 & 1 & 0.421 \\
\hline
\end{tabular}


Similarly, from univariate analysis of each feature derived from DST raw data (shown in Table 5), the handwriting features found to be most correlated to PD from DST are: peak instantaneous acceleration (PIA), standard deviation of grip angle (GASD), average pressure (PMean), increase in pressure (PCMax) and height of the drawing output (Height).

It must be noted that the recall value for the height variable control condition is lower than the threshold, however, it was considered as borderline significant as a $5^{\text {th }}$ variable for consistency with the SST set.

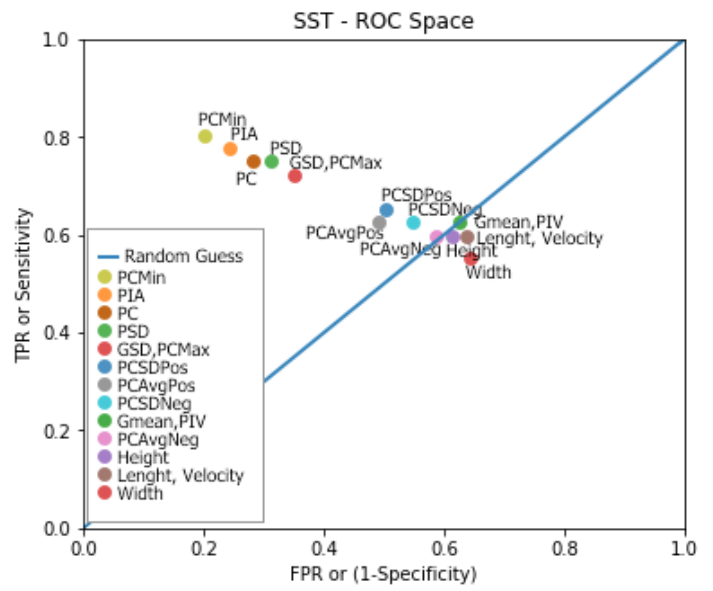

Figure 3. ROC plot showing the relative predictive potential of SST handwriting features

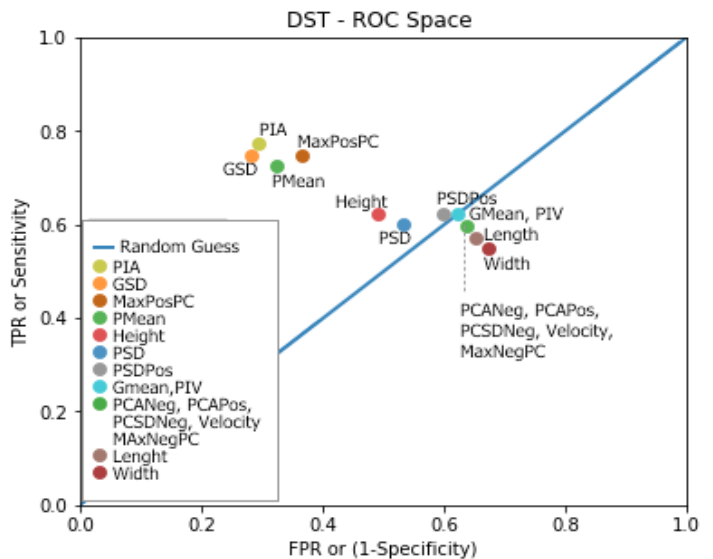

Figure 4. ROC plot showing the relative predictive potential of DST handwriting features

Receiver Operating Curve (ROC) space plots were then generated to visualize the relative significance of the features for each test type (Figure 3 and Figure 4). The values directly correspond to their significance ranking in Table 4 and Table 5 and indicate differential power, with the most correlative handwriting features closest to $\mathrm{TPR}=1$ and $\mathrm{FPR}=0$, i.e. towards the top left corner of the chart.

\subsection{Classification Model Generation \& Evaluation}

The most significant handwriting features (highlighted in Table 4 and Table 5) were then selected to build multivariate classification models for each test type (SST and DST). Logistic regression models with 10 -fold cross validation were constructed firstly with the top 5 most significant features from univariate analysis, then iteratively refined by following a stepwise backward elimination approach, tested for multicollinearity and assessed based on the classification accuracy of the models. The results are presented as follows.

\subsubsection{SST}

A logistic regression model based on the 5 most significant SST features obtained from univariate analysis (PressureSD, GripangleSD, PressureChangeMax, PressureChangeMin, PeakInstantaneousAcceleration) yielded a classification accuracy of $90 \%$.

\section{Table 6. SST based model (5-variables)}

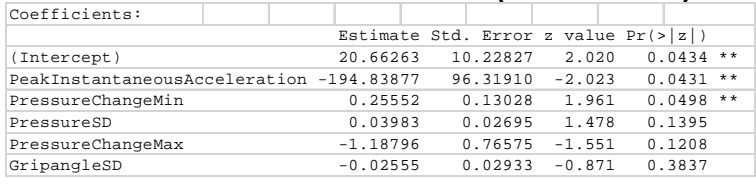

As shown in Table 6, GripangleSD was found to be the least statistically significant. Elimination of this variable was found to improve classification accuracy to $92.5 \%$. Exclusion of the next less significant variable, PressureSD, yielded a combined classification accuracy of $85 \%$, indicating that classification accuracy peaked at $92.5 \%$ with 4 variables ( Table 7).

\section{Table 7. SST final model (4-variables)}

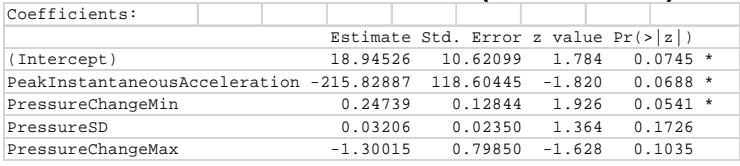

Further elimination and inclusion of other variables was not found to improve upon this value. These results indicate that the significant features for the SST test in differentiating PD are: peak instantaneous acceleration, maximum increase in pressure, maximum decrease in pressure and standard deviation of pressure. 
An ROC Space plot for the SST multivariate logistic regression models (5-variable, 4-variable and 3varaible) was generated to visualize the relative classification performances (Figure 5).

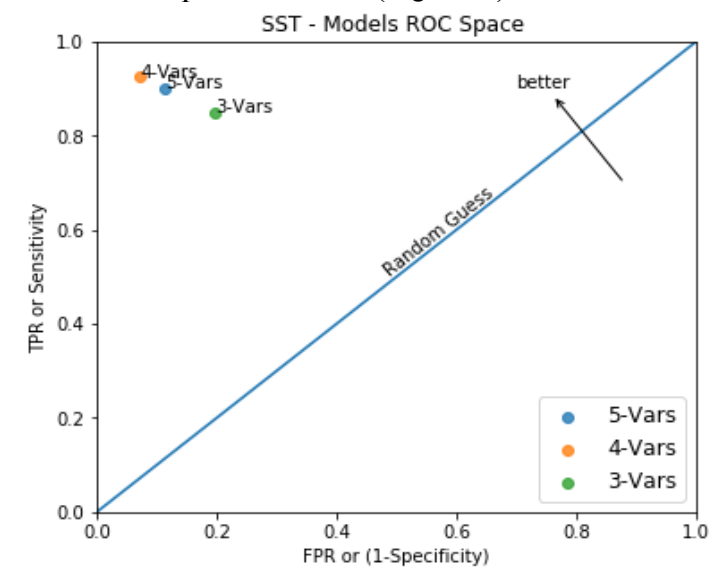

Figure 5. ROC Space plot showing the relative performance of the SST classification model

\subsubsection{DST}

The combined classification accuracy from multivariate analysis of the 5 most significant DST features (PeakInstantaneousAcceleration, GripangleSD, PressureChangeMax, PressureMean and Height) was found to be $85 \%$.

PressureMean was found to be the least statistically significant feature (Table 8), and its exclusion from the model yielded a combined classification accuracy of $85 \%$, confirming that it is independently insignificant.

Stepwise exclusion of PresureMean (4-variable model: GASD, PIA, PCMax, Height) and PCMax (3variable model: GASD, PIA, Height) was found to also yield classification accuracies of $85 \%$. This result is as expected given the similarity in significance ranking of Height and PressureSD from univariate analysis. From multivariate analysis, PressureSD was found to hold higher differential significance than PressureMean as a pressure index

Removal of Height from the feature set reduced classification accuracy to $77.5 \%$, indicating that it has associative significance with other handwriting features. Collinearity can be expected as these features were calculated from the same base raw variables

Table 8. DST based model (5-variables)

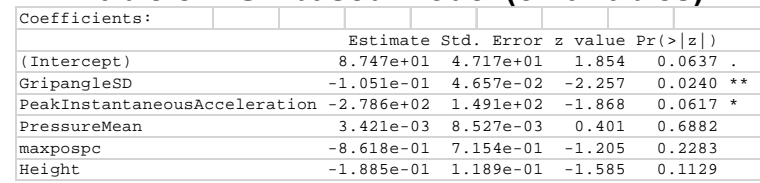

These results indicate that that the significant features for the DST test in differentiating PD are: peak instantaneous acceleration, variation in grip angle and height of the drawing output (Table 9).

\section{Table 9. DST final model (3-variables)}

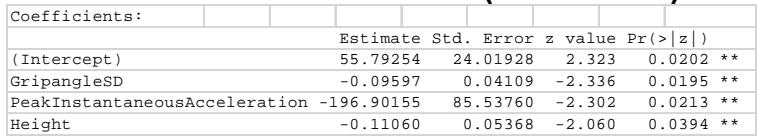

An ROC Space plot for the multivariate logistic regression (5-variable, 4-variable and 3-varaible) for DST with 10 -fold cross validation were generated to visualize the relative classification performance of each model (Figure 6).

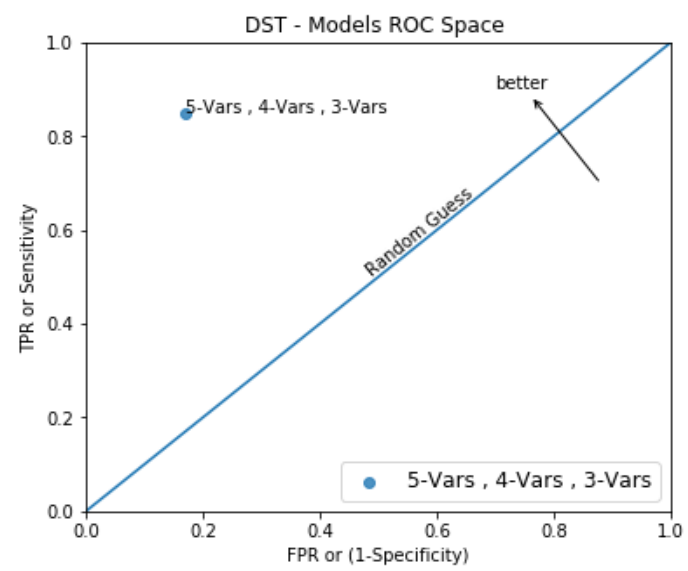

Figure 6. ROC Space plot showing the relative performance of DST classification model

\section{Discussion \& Clinical Interpretation}

\subsection{Feature expansion \& analysis}

Given that the shift towards digitized handwriting analysis in PD research is a relatively recent development, feature expansion is an important step to explore and derive potentially informative kinematic handwriting features that have not been previously considered. Indeed, secondary handwriting features obtained from the feature expansion step in this study were shown to have high predictive potential. Classification models based on a combination of the most significant features from 2-step logistic regression was found to yield high classification accuracies of $85 \%$ and $92.5 \%$ for DST and SST drawing tasks respectively. 
All 4 final SST model variables were found to be secondary handwriting features, whereas 2 of 3 the final DST model variables were secondary. These results validate the feature expansion step and consideration of latent kinematic characteristics in the analysis of PD handwriting.

\subsection{Comparison of Static and Dynamic Spiral Tests}

From a biomechanical perspective, it can be assumed that similar general sequences of hand, wrist, and upper limb motion are required to produce the same geometrical output. As both the static and dynamic Spiral Drawing Tests involve tracing the same Archimedean spiral template, it follows that the correlative ranking of individual features will be highly similar for both tests, and that any differences in feature significance are informative of the effect of the additional cognitive and temporal components of the DST on drawing motion within the respective experimental groups.

Our results revealed differences in the statistical significance of individual handwriting features between the SST and DST. Overall, the additional testing conditions introduced by the DST resulted in greater variability and lower classification accuracy of individual features and in combination compared to results obtained for the SST. Further investigation with a larger experimental group is required to more definitively determine the influence of DST conditions compared to SST.

It can be seen that the SST was more sensitive to assessing reduction and variations in pressure, which is consistent with previous reports of reduced writing pressure in PD groups [11] whereas the DST resulted in greater variations in grip angle and increased pressure. This is likely due to greater cognitive load required to trace the spiral template and heightened stress from altered visual-motor feedback and perception of time due to the blinking template.

Peak instantaneous acceleration, a measurable manifestation of PD tremor, was among the highest correlative indicator of PD for both test types, suggesting its reliability as a clinical test biomarker.

Interestingly, the height of the DST drawing output was found to have predictive significance but less so for the SST. It is possible that the temporal and visualmotor feedback factors introduced by the DST exacerbate micrographia. PD patients have been reported to draw cursive loops progressively smaller with repetition [9]. As spiral drawing involves similar looped motion, it is possible that as both SST and DST test types were administered consecutively in the original study, the DST data was collected after the SST and was impacted by neuromuscular fatigue from repeated testing, which in turn emphasized micrographia in PD study participants. Further study where patients perform the SST and DST in individual sittings or with an adequate resting phase between data collection is required to investigate the phenomenon and elucidate comparison of the test types.

\subsection{Correlation of Handwriting Features with Parkinson's Disease Neuropathology}

Tremor, micrographia, rigidity and bradykinesia (slowness of movement) are well recognized to be early manifestations of PD [4]. The results of our preliminary analysis indicate that peak instantaneous acceleration, pressure and grip indices hold high predictive potential as markers of PD in spiral drawing tasks. These findings are consistent with other reports that PD patients display greater variations in writing pressure [10]. The association between handwriting features analyzed and established clinical presentations of PD are summarized in Table 10.

Acceleration is a kinematic measure of jerk due to tremor, a clinical hallmark of Parkinson's Disease. Other related features to tremor include variations in applied pressure. The dynamic spiral test was shown to accentuate reduced drawing height, which is consistent with micrographia, more than the static spiral test. Further study is required to determine the role of visual-motor feedback in completion of digitized drawing tasks.

Table 10. Correlation between handwriting features and Parkinson's Disease symptoms PD hallmark Corresponding handwriting presentation features \& abnormalities

Tremor Peak instantaneous acceleration, peak instantaneous velocity, jerk, variations in applied pressure, absolute drawing output length

Bradykinesia Writing speed, micrographia, (slowness of deviation from template, height movement) and length of drawing output

Rigidity Writing speed, micrographia, grip, deviation from template, graphical features of drawing output, variations in applied pressure, average pressure

Dementia Deviation from (correlated template/difficulty following task, with latter variations in applied pressure, stage PD) drawing output dimensional variations 


\section{Conclusions}

In the current diagnostic regime for $\mathrm{PD}$, a combination of several drawing tasks is typically performed as the shape and design of each drawing task recruits different sets of cognitive and fine motor functions relevant to diagnosis of $\mathrm{PD}$, e.g. drawing a circle differs from the angular motions required for producing a pentagon. The selection of tasks varies between studies, depending on the diagnosis the task is designed to assess, and the judgement of the clinician. Digitization of established drawing tests such as the spiral drawing test will allow objective comparison and evaluation of their clinicometric relevance in addition to their diagnostic value.

Our study explored and analyzed previously unconsidered secondary handwriting features in the dataset from Isenkul et al's Standard Spiral Test and Dynamic Spiral Test study. Our results demonstrate that secondary handwriting features, particularly changes in pressure indices, hold predictive potential in the differential diagnosis of Parkinson's Disease from spiral drawing tasks. Feature expansion was shown to be conducive to hypothesis generation. Our systematic approach showed differences in the statistical correlation of individual features between the test types, indicating that the dynamic spiral test is sufficiently different from the standard spiral drawing test to be considered as a new test type. A combination of both static and dynamic tests, along with consideration of the secondary kinematic handwriting features presented in this study, have great potential to improve objective measurement of PD severity and our understanding of the subtle manifestations of PD in handwriting for earlier detection.

Limitations of our findings include the small sample size of the dataset and lack of control in study design and data collection. Further investigation of larger sample sizes is required for greater confidence and confirmation of the differential power of individual handwriting features determined by our analysis.

Furthermore, as digitization represents a paradigm shift of cognitive drawing tasks to a new platform, research is required in the development of a standardized protocol, tools and new drawing tests such as the Dynamic Spiral Test to optimally harness the capabilities of digital analysis, as well as to extend the application to assessing interrelated cognitive factors e.g. reflexes and memory. Task-dependent indices will also require development to allow meaningful comparison and evaluation of drawing tasks.

\section{Conflict of Interest}

The authors have no conflict of interest to declare.

\section{References}

[1] Tysnes, O.-B. and Storstein, A. Epidemiology of Parkinson's disease. Journal of Neural Transmission, 124, 8 (August 01 2017), 901-905.

[2] Sveinbjornsdottir, S. The clinical symptoms of Parkinson's disease. Journal of Neurochemistry, 139 (2016), 318-324.

[3] Contreras-Vidal, J. and Stelmach, G. E. Effects of parkinsonism on motor control. Life Sciences, 58, 3 (1995/12/08/ 1995), 165-176.

[4] Jankovic, J. Parkinson's disease: clinical features and diagnosis. Journal of Neurology, Neurosurgery \&amp; Psychiatry, 79, 4 (2008), 368-376.

[5] Goetz, C. G., Tilley, B. C., Shaftman, S. R., Stebbins, G. T., Fahn, S., Martinez-Martin, P., Poewe, W., Sampaio, C., Stern, M. B., Dodel, R., Dubois, B., Holloway, R., Jankovic, J., Kulisevsky, J., Lang, A. E., Lees, A., Leurgans, S., LeWitt, P. A., Nyenhuis, D., Olanow, C. W., Rascol, O., Schrag, A., Teresi, J. A., van Hilten, J. J. and LaPelle, N. Movement Disorder Society-sponsored revision of the Unified Parkinson's Disease Rating Scale (MDS-UPDRS): Scale presentation and clinimetric testing results. Movement Disorders, 23, 15 (2008), 2129-2170.

[6] Goetz, C. G., Poewe, W., Rascol, O., Sampaio, C., Stebbins, G. T., Counsell, C., Giladi, N., Holloway, R. G., Moore, C. G., Wenning, G. K., Yahr, M. D. and Seidl, L. Movement Disorder Society Task Force report on the Hoehn and Yahr staging scale: Status and recommendations The Movement Disorder Society Task Force on rating scales for Parkinson's disease. Movement Disorders, 19, 9 (2004), 1020-1028.

[7] Pahwa, R. and Lyons, K. E. Early diagnosis of Parkinson's disease: recommendations from diagnostic clinical guidelines. Am J Manag Care, 16 Suppl Implications (2010/03// 2010), S94-99.

[8] Tolosa, E., Wenning, G. and Poewe, W. The diagnosis of Parkinson's disease. The Lancet Neurology, 5 , 1 (2006/01/01/ 2006), $75-86$

[9] Yu, N.-Y., Van Gemmert, A. W. A. and Chang, S.-H. Characterization of graphomotor functions in individuals with Parkinson's disease and essential tremor. Behavior research methods, 49, 3 (June 01 2017), 913-922.

[10] Drotár, P., Mekyska, J., Rektorová, I., Masarová, L., Smékal, Z. and Faundez-Zanuy, M. Evaluation of handwriting kinematics and pressure for differential diagnosis of Parkinson's disease. Artificial Intelligence in Medicine, 67 (2016/02/01/ 2016), 39-46.

[11] Rosenblum, S., Samuel, M., Zlotnik, S., Erikh, I. and Schlesinger, I. Handwriting as an objective tool for Parkinson's disease diagnosis. Journal of Neurology, 260, 9 (September 2013 2013), 2357-2361.

[12] Smits, E. J., Tolonen, A. J., Cluitmans, L., Van Gils, M., Conway, B. A., Zietsma, R. C., Leenders, K. L. and Maurits, 
N. M. Standardized handwriting to assess bradykinesia, micrographia and tremor in Parkinson's disease. PloS one, 9, 5 (2014).

[13] Drotár, P., Mekyska, J., Rektorová, I., Masarová, L., Smékal, Z. and Faundez-Zanuy, M. Decision Support Framework for Parkinson's Disease Based on Novel Handwriting Markers. IEEE Transactions on Neural Systems and Rehabilitation Engineering, 23, 3 (2015), 508-516.

[14] Pirlo, G., Diaz, M., Ferrer, M. A., Impedovo, D., Occhionero, F. and Zurlo, U. Early Diagnosis of Neurodegenerative Diseases by Handwritten Signature Analysis. Springer International Publishing, City, 2015.

[15] Kawa, J., Bednorz, A., Stępień, P., Derejczyk, J. and Bugdol, M. Spatial and dynamical handwriting analysis in mild cognitive impairment. Computers in Biology and Medicine, 82 (2017/03/01/ 2017), 21-28.

[16] Kaul, S. and Elble, R. J. Impaired pentagon drawing is an early predictor of cognitive decline in Parkinson disease. Movement disorders : official journal of the Movement Disorder Society, 29, 03 (01/21 2014), 427-428.

[17] Nagaratnam, N., Nagaratnam, K. and O'Mara, D. Intersecting pentagon copying and clock drawing test in mild and moderate Alzheimer's disease. Journal of Clinical Gerontology and Geriatrics, 5, 2 (2014/06/01 2014), 47-52.

[18] Cormack, F., Aarsland, D., Ballard, C. and Tovée, M. J. Pentagon drawing and neuropsychological performance in Dementia with Lewy Bodies, Alzheimer's disease, Parkinson's disease and Parkinson's disease with dementia. International Journal of Geriatric Psychiatry, 19, 4 (2004), 371-377.

[19] Müller, S., Preische, O., Heymann, P., Elbing, U. and Laske, C. Increased Diagnostic Accuracy of Digital vs. Conventional Clock Drawing Test for Discrimination of Patients in the Early Course of Alzheimer's Disease from Cognitively Healthy Individuals. Frontiers in Aging Neuroscience, 9 (2017), 101.

[20] Wang, M., Wang, B., Zou, J., Chen, L., Shima, F. and Nakamura, M. A new quantitative evaluation method of Parkinson's disease based on free spiral drawing. City, 2010.

[21] Isenkul, M., Sakar, B. and Kursun, O. Improved Spiral Test Using Digitized Graphics Tablet for Monitoring Parkinson's Disease, 2014.

[22] Stanley, K., Hagenah, J., Brüggemann, N., Reetz, K., Severt, L., Klein, C., Yu, Q., Derby, C., Pullman, S. and Saunders-Pullman, R. Digitized Spiral Analysis is a Promising Early Motor Marker for Parkinson Disease. Parkinsonism \& related disorders, 16, 3 (01/15 2010), 233234.

[23] Yu, N.-Y. and Chang, S.-H. Kinematic Analyses of Graphomotor Functions in Individuals with Alzheimer's Disease and Amnestic Mild Cognitive Impairment. Journal of Medical and Biological Engineering, 36, 3 (2016/06/01 2016), 334-343.
[24] Smits, E. J., Tolonen, A. J., Cluitmans, L., van Gils, M., Conway, B. A., Zietsma, R. C., Leenders, K. L. and Maurits, N. M. Standardized Handwriting to Assess Bradykinesia, Micrographia and Tremor in Parkinson's Disease. PLoS ONE, 9, 5 (accepted 2014), e97614.

[25] Teulings, H. L., Contreras-Vidal, J. L., Stelmach, G. E. and Adler, C. H. Adaptation of handwriting size under distorted visual feedback in patients with Parkinson's Disease and elderly and young controls. Journal of Neurology, Neurosurgery \& Psychiatry, 72, 3 (2002), 315.

[26] Nackaerts, E., Heremans, E., Smits-Engelsman, B. C. M., Broeder, S., Vandenberghe, W., Bergmans, B. and Nieuwboer, A. Validity and reliability of a new tool to evaluate handwriting difficulties in Parkinson's disease. PLOS ONE, 12, 3 (2017), e0173157.

[27] Ünlü, A., Brause, R. and Krakow, K. Handwriting Analysis for Diagnosis and Prognosis of Parkinson's Disease. In Proceedings of the Biological and Medical Data Analysis: 7th International Symposium, ISBMDA 2006, Thessaloniki, Greece, December 7-8, 2006. Proceedings (Berlin, Heidelberg, 2006//, 2006). Springer Berlin Heidelberg, [insert City of Publication],[insert 2006 of Publication].

[28] Goodnow, J., Friedman, S. L., Bernbaum, M. and Lehman, E. B. Direction and Sequence in Copying:The Effect of Learning to Write in English and Hebrew. Journal of Cross-Cultural Psychology, 4, 3 (1973), 263-282.

[29] Kraus, P. H. and Hoffmann, A. Spiralometry: Computerized assessment of tremor amplitude on the basis of spiral drawing. Movement Disorders, 25, 13 (2010), 21642170 .

[30] Drotár, P., Mekyska, J., Smékal, Z., Rektorová, I., Masarová, L. and Faundez-Zanuy, M. Prediction potential of different handwriting tasks for diagnosis of Parkinson's. City, 2013.

[31] Hoogendam, Y. Y., van der Lijn, F., Vernooij, M. W., Hofman, A., Niessen, W. J., van der Lugt, A., Ikram, M. A. and van der Geest, J. N. Older Age Relates to Worsening of Fine Motor Skills: A Population-Based Study of MiddleAged and Elderly Persons. Frontiers in Aging Neuroscience, 6, 259 (2014-September-25 2014).

[32] Thomas, M., Lenka, A. and Kumar Pal, P. Handwriting Analysis in Parkinson's Disease: Current Status and Future Directions. Movement Disorders Clinical Practice, 4, 6 (2017), 806-818.

[33] Rosenblum, S., Parush, S. and Weiss, P. L. Computerized Temporal Handwriting Characteristics of Proficient and Non-Proficient Handwriters. American Journal of Occupational Therapy, 57, 2 (2003), 129-138.

[34] Isenkul, M. and Sakar, B. Parkinson Disease Spiral Drawings Using Digitized Graphics Tablet Data Set City, 2017. 